\title{
An automated fluorescence videomicroscopy assay for the detection of mitotic catastrophe
}

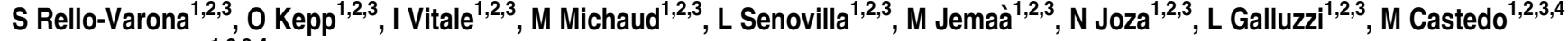 \\ and G Kroemer ${ }^{\star, 1,2,3,4}$
}

Mitotic catastrophe can be defined as a cell death mode that occurs during or shortly after a prolonged/aberrant mitosis, and can show apoptotic or necrotic features. However, conventional procedures for the detection of apoptosis or necrosis, including biochemical bulk assays and cytofluorometric techniques, cannot discriminate among pre-mitotic, mitotic and post-mitotic death, and hence are inappropriate to monitor mitotic catastrophe. To address this issue, we generated isogenic human colon carcinoma cell lines that differ in ploidy and p53 status, yet express similar amounts of fluorescent biosensors that allow for the visualization of chromatin (histone H2B coupled to green fluorescent protein (GFP)) and centrosomes (centrin coupled to the Discosoma striata red fluorescent protein (DsRed)). By combining high-resolution fluorescence videomicroscopy and automated image analysis, we established protocols and settings for the simultaneous assessment of ploidy, mitosis, centrosome number and cell death (which in our model system occurs mainly by apoptosis). Time-lapse videomicroscopy showed that this approach can be used for the high-throughput detection of mitotic catastrophe induced by three mechanistically distinct anti-mitotic agents (dimethylenastron (DIMEN), nocodazole (NDZ) and paclitaxel (PTX)), and - in this context - revealed an important role of p53 in the control of centrosome number.

Cell Death and Disease (2010) 1, e25; doi:10.1038/cddis.2010.6; published online 18 February 2010

Subject Category: Cancer

This is an open-access article distributed under the terms of the Creative Commons Attribution License, which permits distribution and reproduction in any medium, provided the original author and source are credited. This license does not permit commercial exploitation without specific permission.

The deregulation of cell death is quintessential for the pathogenesis of numerous human diseases. Therefore, the in-depth analysis of the cellular demise and its mechanisms constitutes a major challenge for fundamental and applied biomedical research at the same time as it conditions the development of novel therapeutic interventions. ${ }^{1-4}$ Programmed cell death can be mediated by distinct mechanisms, including apoptosis and necrosis, and can also be associated with the massive activation of autophagy.,6 Hundreds of methods are available for the detection of these phenomena and their biochemical correlates in cellular extracts, cultured cells, isolated tissues as well as in living organisms, each characterized by specific advantages and pitfalls. ${ }^{7-9}$

Mitotic catastrophe has originally been described as 'an event of abnormal mitosis that makes it impossible for cells to proliferate, regardless of the mechanism or the timing'. ${ }^{10}$ Although the so-called 'delayed radioinduced cell death', which similarly reflects post-mitotic death, can take place years after radiotherapy in human tissues, ${ }^{11}$ it may be useful to identify mitotic catastrophe in a more pragmatic way, by referring to a specific (and hence surveyable) kinetics. Along these lines, mitotic catastrophe has been defined as an event of apoptotic or necrotic cell death that occurs during or shortly after an aberrant, often protracted mitosis. ${ }^{12,13}$ More precisely, mitotic catastrophe should be regarded as a process that can lead to a number of different outcomes, depending on the trigger and the cellular context. ${ }^{13,14}$ As a matter of fact, not only mitotic catastrophe interfaces with cell death through apoptosis but also with necrosis, senescence and viable polyploidization, either in developmental conditions and in response to anticancer treatments. ${ }^{13,15-17}$ Moreover, recent publications point to the capacity of aneuploid cells to undergo de-polyploidization by multipolar division, ${ }^{18,19}$ a phenomenon that might lead to the generation of aneuploid cancer stem cells. ${ }^{20}$

Mitotic catastrophe frequently involves the apical activation of one particular caspase, caspase-2. ${ }^{15}$ However, as caspase-2 can be activated by mitosis-unrelated stimuli (e.g., DNA damage, Golgi apparatus stress and heat shock) $)^{21-24}$ and, in some instances, mitotic catastrophe proceeds through caspase-2-independent mechanisms, ${ }^{13,17,25}$ the standalone

\footnotetext{
${ }^{1}$ INSERM U848, Villejuif F-94805, France; ${ }^{2}$ Institut Gustave Roussy, Villejuif F-94805, France and ${ }^{3}$ Université de Paris XI-Paris Sud, Villejuif F-94805, France *Corresponding author: G Kroemer, INSERM U848, Institut Gustave Roussy, Pavillon de Recherche 1, 39 rue Camille Desmoulins, Villejuif F-94805, France. Tel: + 3314211 6046; Fax: + 3314211 6047; E-mail: kroemer@ orange.fr

${ }^{4}$ These authors share senior co-authorship.

Keywords: mitotic catastrophe; centrosome; multipolar mitosis; apoptosis; p53; cell death detection

Abbreviations: AU, arbitrary unit; DIMEN, dimethylenastron; DsRed, Discosoma striata red fluorescent protein; FACS, fluorescence-activated cell sorter; FSC, forward scatter; GFP, green fluorescent protein; H2B, histone H2B; NDZ, nocodazole; PTX, paclitaxel; PI, propidium iodide; ROI, region of interest; S.E.M., standard error of the mean; SSC, side scatter; STS, staurosporine; UCN-01, 7-hydroxystaurosporine; WT, wild type

Received 12.1.10; accepted 15.1.10; Edited by G Melino
} 
assessment of caspase-2 activity does not allow for the accurate identification of this cell death subroutine.

Mitotic catastrophe can result from endogenous causes. For example, polyploid cells often engage in abnormal mitosis due to the presence of supernumerary centrosomes, lagging chromosomes and/or accumulated unrepaired DNA lesions. ${ }^{26,27}$ Most of the cells that enter aberrant mitosis finally die, probably because of checkpoint mechanisms aiming to minimize the risk of genomic instability. In line with this hypothesis, we have previously shown that the inhibition of mitotic catastrophe increases the propensity of cells to enter multipolar, asymmetric divisions that eventually lead to aneuploidy. ${ }^{12}$ In addition, mitotic catastrophe can be induced by exogenous agents including ionizing irradiation, ${ }^{28}$ microtubule poisons, ${ }^{29}$ inhibitors of the mitotic kinesin $E g 5^{30}$ as well as by pharmacological/genetic strategies that specifically target the checkpoint kinase Chk1. ${ }^{31}$ However, the percentage of cells that succumb to mitotic catastrophe as opposed to classical interphase apoptosis in response to microtubule poisons depends on the cellular context. ${ }^{32}$ The competition between the molecular machinery for caspase activation and the regulators of the abundance of cyclin B1 (which exerts an effect as an inhibitor of mitotic slippage) determines whether cells will die during interphase or will succumb to mitotic catastrophe. $^{32}$

Conventional methods for the detection of cell death cannot trace the 'history' of the cellular demise with respect to mitosis. Apoptotic and necrotic markers, including chromatin condensation, phosphatidylserine exposure, cytochrome $c$ release from mitochondria and plasma membrane permeabilization, can all occur before, during or after mitosis. ${ }^{33-35}$ Moreover, the end-stage products of apoptosis or necrosis in post-mitotic and proliferating cells are indistinguishable. ${ }^{7}$ Therefore, none of the methods that are routinely used for the detection of cell death is applicable for the accurate quantification of mitotic catastrophe. At present, the only approach for the assessment of mitotic catastrophe relies on the continuous observation of cells by conventional microscopy, which is tedious, time consuming and operator dependent. To circumvent the intrinsic problems of conventional microscopy-based assays, automated high-content image-based methods have to be developed. ${ }^{36,37}$ Driven by these considerations, we decided to implement an automated videomicroscopy assay for the real-time, simultaneous detection of mitosis, ploidy, supernumerary centrosomes and cell death that can be used for the high-throughput detection of mitotic catastrophe.

\section{Results and Discussion}

Automatic detection of mitotic arrest. Wild-type (WT) and p53-deficient $\left(p 53^{-/-}\right)^{38}$ human colon carcinoma HCT 116 cells had previously been engineered for the stable expression of a histone H2B-green fluorescent protein (H2B-GFP) chimera, allowing for the visualization of chromatin and hence for the continuous tracking of chromosomal movement. $^{16,39}$ To simultaneously monitor the centrosome cycle, we further transfected these cells with a plasmid encoding centrin, an essential constituent of centrosomes, fused to the C-terminus of a Discosoma striata red fluorescent protein variant (DsRed-Centrin chimera) (Supplementary Figure 2). Stable clones expressing moderate amounts of the red fluorescent protein in dot-like structures (mostly one single dot per cell, corresponding to the centrosome) were selected, and videomicroscopy was used to confirm normal DNA replication and centrosome cycle (Figure 1a). The presence of DsRed-Centrin neither affected the growth rate of WT and $p 53^{-/-}$HCT 116 cells (Figure $1 \mathrm{~b}$ ) nor their cell cycle distribution (Figure 1c). Upon nocodazole (NDZ)-mediated tetraploidization (Supplementary Figure 1) and selection of stable WT and $p 53^{-/-}$tetraploid clones, ${ }^{31,39}$ the expression of DsRed-Centrin remained constant, thereby allowing for the detection of supernumerary centrosomes (Figure 1d), which are known to arise in $p 53^{-/}$tetraploid cells. ${ }^{40}$

We systematically exposed WT diploid, $p 53^{-/-}$diploid, WT tetraploid and $p 53^{-/-}$tetraploid HCT cells to three distinct inducers of mitotic catastrophe, namely paclitaxel (PTX, a taxane that irreversibly inhibits microtubular depolymerization), ${ }^{41}$ dimethylenastron (DIMEN, which targets the mitotic kinesin Eg5) ${ }^{30}$ and NDZ (a reversible inhibitor of microtubular polymerization), ${ }^{42}$ as well as to 7-hydroxystaurosporine (UCN-01), a staurosporine (STS) derivative that induces apoptosis by exerting an effect as a rather unspecific tyrosine kinase inhibitor. ${ }^{43}$ All these chemicals exerted strong cytotoxic/antiproliferative effects, thereby causing a considerable decrease in the frequency of surviving cells irrespective of ploidy and $p 53$ status, as determined $72 \mathrm{~h}$ after administration by automated cell counting in the processed micrographs (Figures 2a and b) and confirmed by the assessment of residual proliferation with a colorimetric tetrazolium salt (WST-1) conversion assay (Figure 2c). It is noteworthy that the diploid cells that survived the administration of mitotic catastrophe inducers (i.e., PTX, DIMEN and NDZ) showed a net increase in nuclear size, presumably because of polyploidization (further discussed below), which is less evident in the fraction of cells that resisted to UCN-01 (Figure 2a).

We next established a procedure for the automated, fluorescence microscopy-based identification of cells arrested in mitosis upon short-term $(8 \mathrm{~h})$ exposure to different mitotic inhibitors and apoptosis inducers (Figure 3a). To foreclose most events of advanced apoptosis (which leads to pronounced nuclear shrinkage and formation of apoptotic bodies, Supplementary Figure 3$)^{5}$ from the analysis, regions of interest (ROIs) characterized by a H2B-GFP signal (used to define the nuclear area) $<100$ pixels were disregarded. Two different parameters were used to identify and quantify mitotic cells within micrographs. The first was granularity, a texture parameter that represents the heterogeneity of the fluorescent signal within a ROI. This was calculated as the ratio between the average difference in fluorescence of adjacent pixels within one ROI and its overall average fluorescence. As in this experimental setting the intensity of fluorescence increases upon H2B-GFP aggregation, granularity provides information on the condensation status of DNA and increases in mitotic or apoptotic cells (Supplementary Figure 3). The other parameter used was the nuclear mean densitometry (that reflects the ratio between the mean fluorescence intensity of the nuclear ROI and its area). By plotting nuclear 
a $\quad 0^{\prime}$

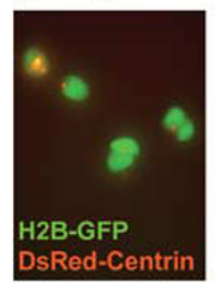

b

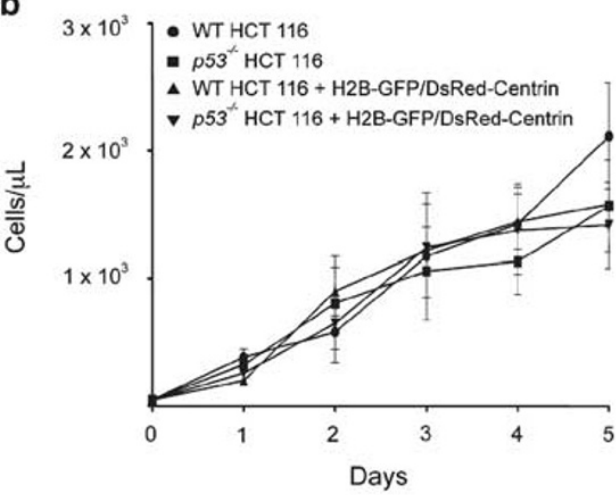

d

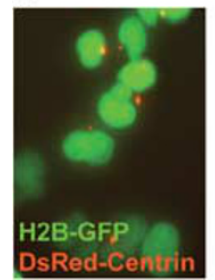

$30^{\prime}$

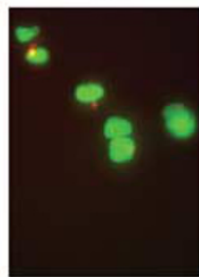

$60^{\prime}$

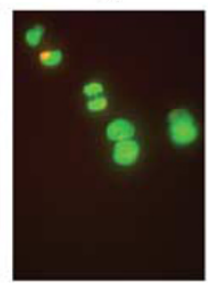

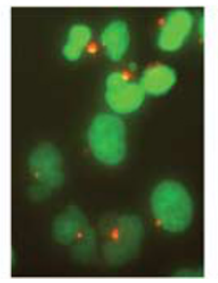

$60^{\prime}$

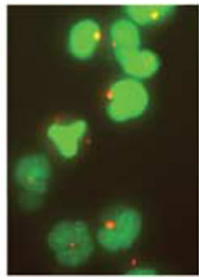

$90^{\prime}$

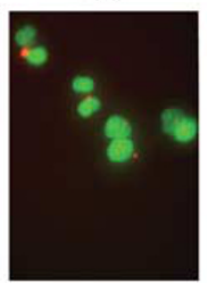

$120^{\prime}$

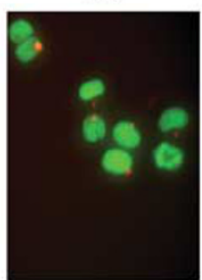

$150^{\prime}$

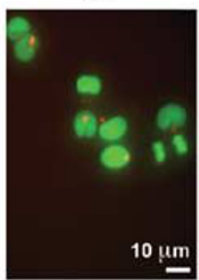

p53 HCT 116

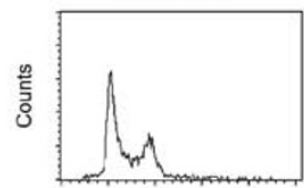

WT HCT 116 H2B-GFP/DsRed-Centrin

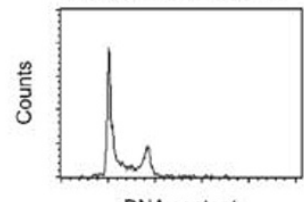
DNA content

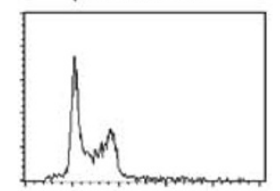

p53. HCT 116 H2B-GFP/DsRed-Centrin

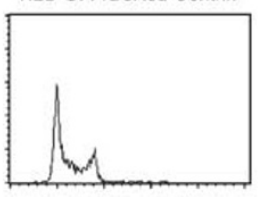

DNA content

$90^{\prime}$

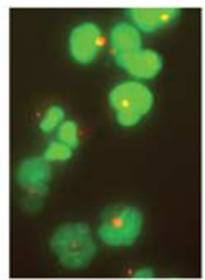

$120^{\prime}$

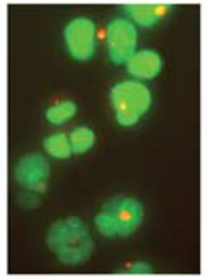

150

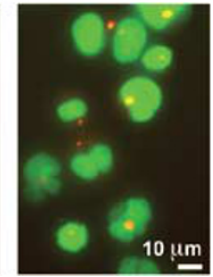

Figure 1 Characterization of human colon cancer HCT 116 cells stably co-expressing H2B-GFP and DsRed-Centrin. (a) Normal cell cycle progression of wild-type (WT) diploid HCT 116 cells engineered for the stable co-expression of H2B-GFP and DsRed-Centrin (which allow for the detection of chromatin status and centrosome number, respectively) was monitored by time-lapse videomicroscopy. Snapshots taken at the indicated time are shown (scale bar $=10 \mu \mathrm{m}$ ). The full-length movie is available as Supplementary Video 1. (b) Hemocytometric cell counting showed that the growth rates of both WT and p53-1- HCT 116 cells co-transfected with H2B-GFP and DsRed-Centrin do not differ from those of their untransfected counterparts. (c) Cytofluorometric analysis upon Hoechst 33342 staining of parental and stably transfected WT and $p 53^{-1-}$ HCT 116 cells confirmed that H2B-GFP/DsRed-Centrin co-expression fails to affect cell cycle distribution. (d) Time-lapse videomicroscopy of $p 53^{-1-}$ tetraploid HCT 116 cells co-expressing H2B-GFP and DsRed-Centrin was used to monitor supernumerary centrosomes, multipolar metaphases and asymmetrical cytokinesis, which reportedly characterize their untransfected counterparts (scale bar $=10 \mu \mathrm{m}$ ). The full-length movie is available as Supplementary Video 2. DsRed, Discosoma striata red fluorescent protein; GFP, green fluorescent protein; H2B, histone H2B

granularity against nuclear mean densitometry, dot plots describing the multiple nuclei belonging to a population of cells could be generated. This allowed us to define threshold levels (and hence gates) for the precise identification of mitotic arrest, in which increased granularity due to chromatin condensation is accompanied by increased mean densitometry. Thus, mitotic cells were defined by granularity of $>550$ arbitrary units (AUs) and mean densitometry of $>250$ AUs (Figure $3 b$ ).

Despite the nuclear area cutoff, the simple comparison between nuclear granularity and nuclear mean densitometry does not allow for the exclusion of all cells that show an apoptotic morphology (as induced by STS). However, as these cells are characterized by increased nuclear granularity as well as by variable densitometry (because of the presence of apoptotic bodies), they show a greater heterogeneity than cells arrested in mitosis, thereby distributing all over the four quadrants of the diagram (which limit their influence on total records; Figure $3 b$ ). This approach allows for not only the identification of cells blocked in metaphase irrespective of its precise morphology, but can also provide some information reflecting the appearance of the mitotic arrest. Thus, typically 'semicircular', 'circular' and 'solid' metaphase plates induced by PTX, DIMEN and NDZ, respectively, are associated with decreasing values of nuclear granularity (Figures $3 \mathrm{~b}$ and $\mathrm{c}$ ). At the same time ( $8 \mathrm{~h}$ ) at which STS results in the accumulation of apoptotic cells, a number of morphologically normal metaphases can be identified upon the administration of UCN-01 (Figure 3c). This is in line with the notion that STS induces apoptosis faster than UCN-01, possibly because it inhibits tyrosine kinases even less specifically than UCN-01 does. ${ }^{43}$ Although all the clones used in this study (irrespective of ploidy and $p 53$ status) responded to (and hence were blocked in metaphase by) PTX, DIMEN and NDZ, the extent of such mitotic arrest was lower in tetraploid cells than in their diploid counterparts. These results were obtained by automated analysis of fluorescence microphotographs (Figure 3d) and confirmed by cytofluorometric assessment of DNA content upon Hoechst 33342 

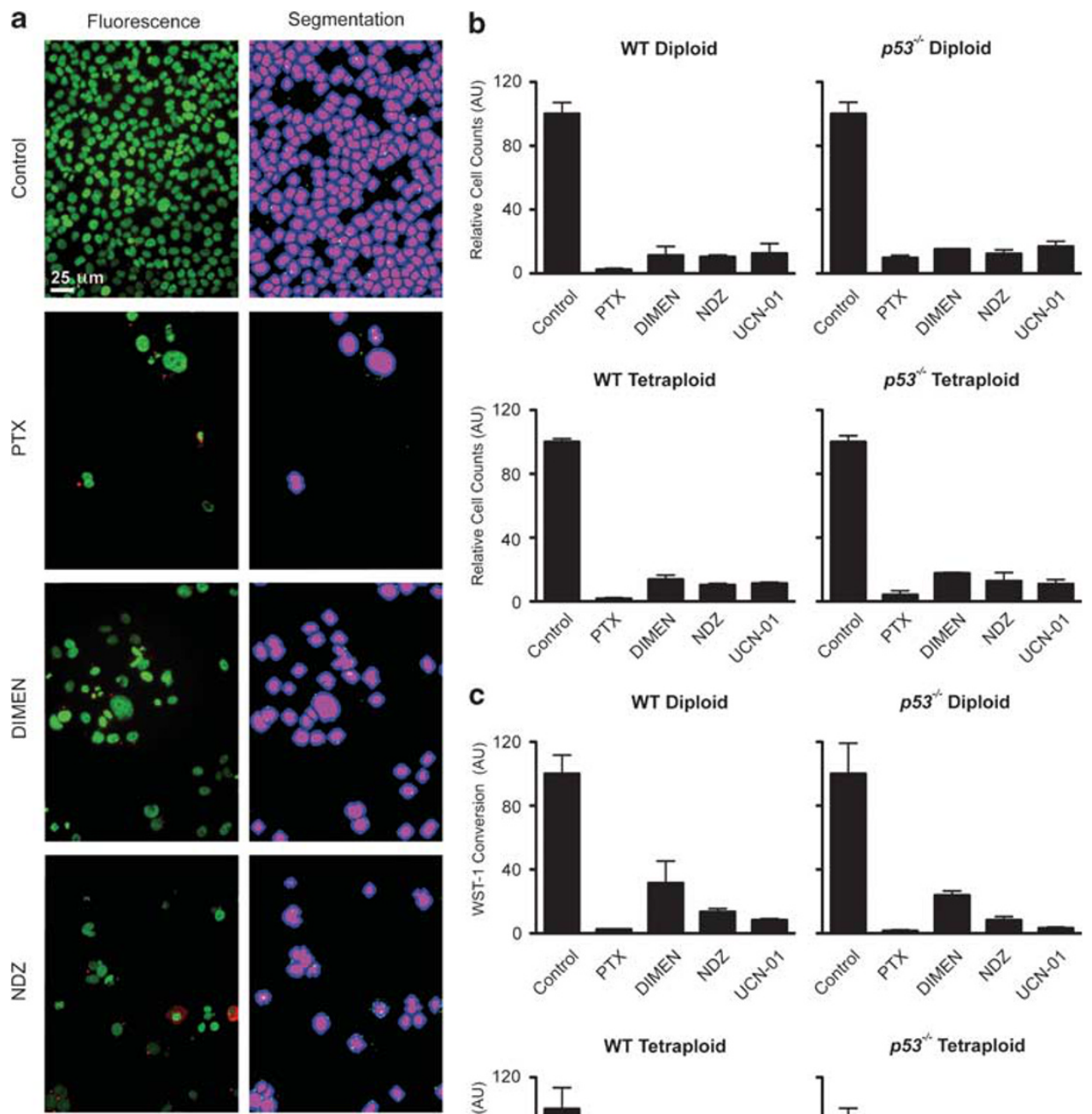

p53 Diploid
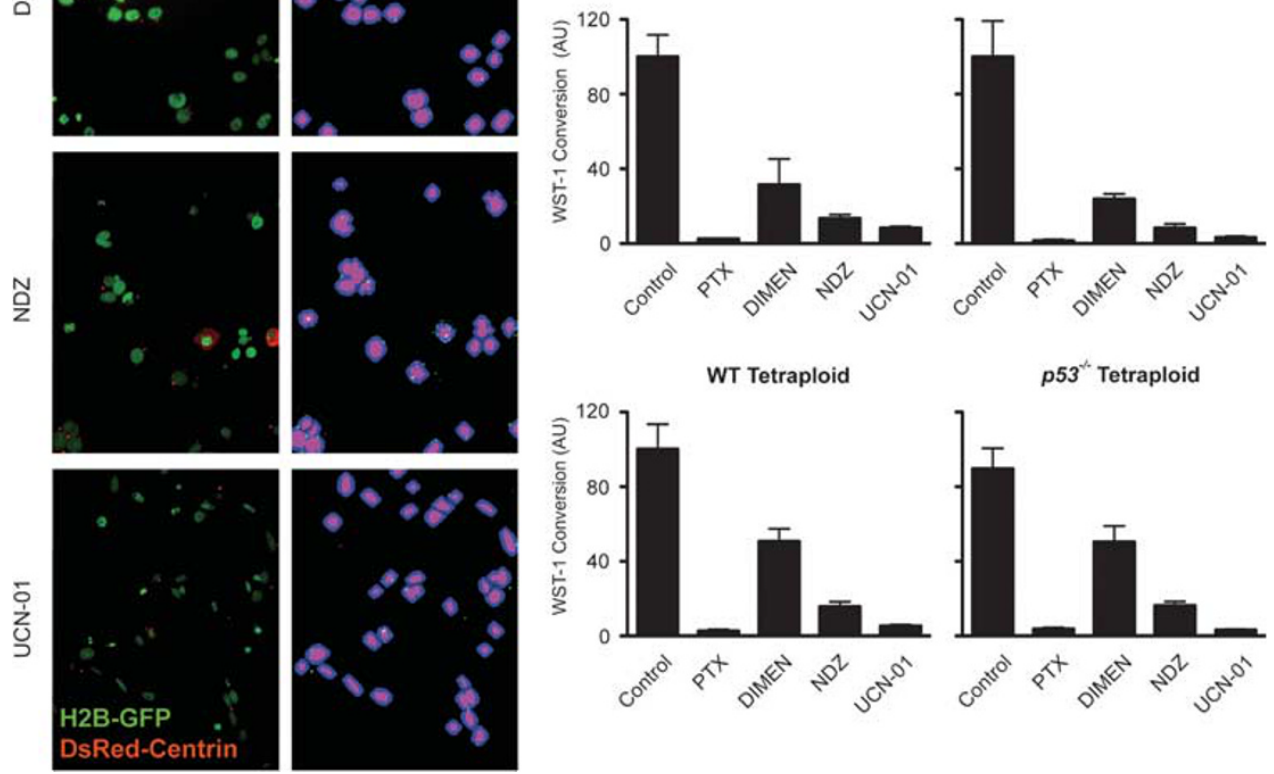

Figure 2 Automated image analysis for the detection of cell death. (a) Fluorescence microphotographs of wild-type (WT) diploid HCT 116 cells co-expressing biosensors for the detection of chromatin status and centrosome number (H2B-GFP and DsRed-Centrin, respectively) - either left untreated (Control) or incubated for $72 \mathrm{~h}$ with paclitaxel (PTX), dimethylenastron (DIMEN), nocodazole (NDZ) or 7-hydroxystaurosporine (UCN-01) - were processed for the creation of the following segmentation masks: nuclei (pink), cells = nuclei +10 pixels (pink + blue); centrosomes (white or green, depending whether they are superposed or not to other regions of interest (ROls)) (scale $\mathrm{bar}=10 \mu \mathrm{m}$ ). (b) Automated cell counting within microphotographs allowed for an indirect assessment of cell survival (untreated cultures were used as a control for the relative estimation of cell number). (c) The indirect quantification of cell survival by colorimetric assays based on the conversion of the tetrazolium salt WST-1 provided similar results. The fraction of surviving cells (WST-1 conversion normalized to the mean of control conditions) is depicted (mean \pm S.E.M., $n=3$ independent experiments). AU, arbitrary unit; DsRed, Discosoma striata red fluorescent protein; GFP, green fluorescent protein; H2B, histone H2B

staining (Figure 3e). Altogether, these data show that fluorescence microscopy can be successfully coupled to automated image analysis for the high-throughput detection of mitotic arrest.

Automatic detection of supernumerary centrosomes and polyploidy. Aberrations of the centrosome cycle often induce multipolar metaphases, which may either occur in a transient manner (followed by congression of supernumerary centrosomes, formation of only two functional microtubule organizing centers and bipolar division) or persist, thereby leading to multipolar cytokinesis. ${ }^{44}$ Thus, we decided to establish one protocol for the automatic detection of the number of centrosomes per cell. In this case, the cell ROI was defined by the nuclear H2B-GFP signal plus a perinuclear extension zone of 10 pixels, and the number of 
a
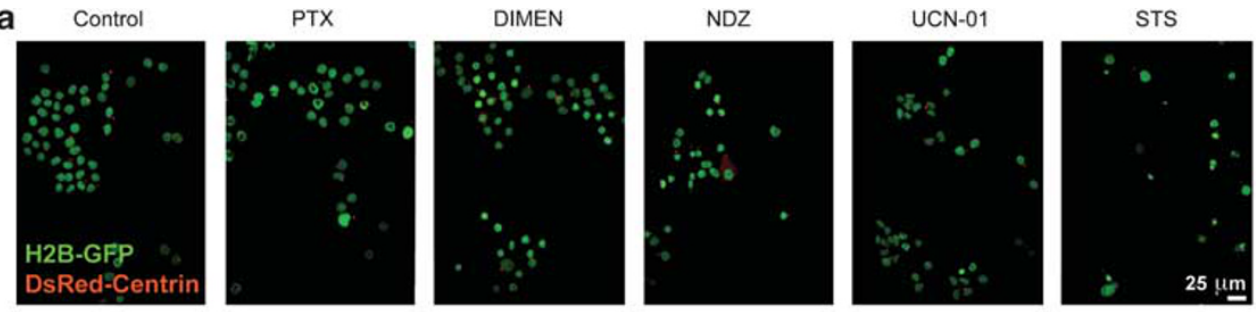

b
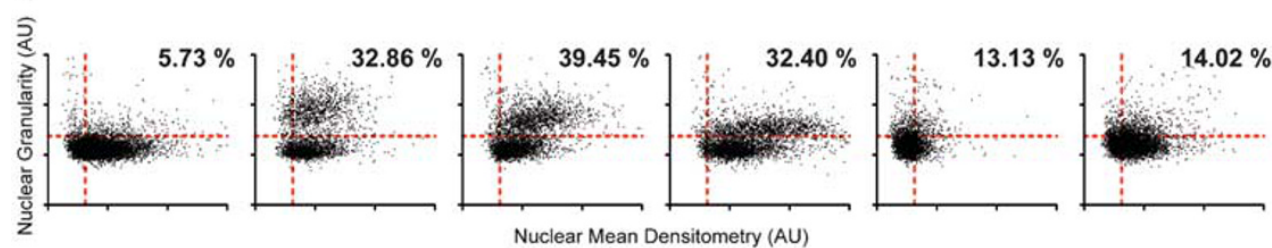

C
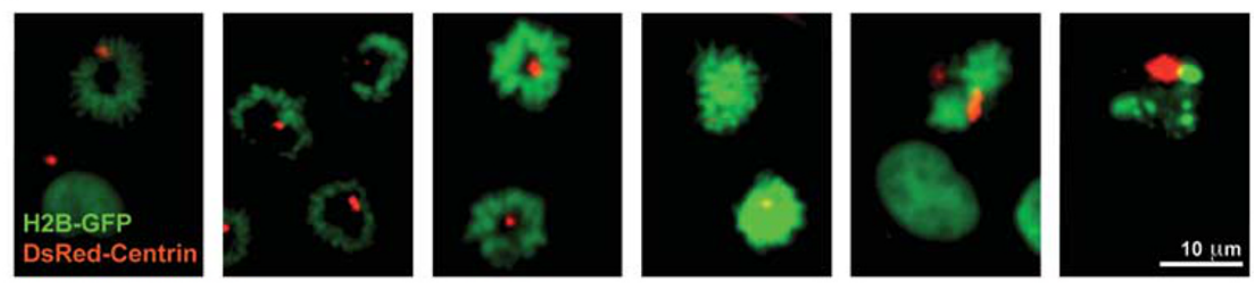

d

WT Diploid

p53\% Diploid
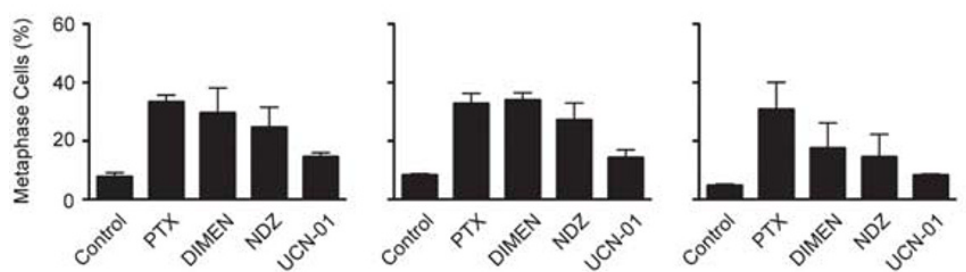

$p 53^{\% /}$ Tetraploid

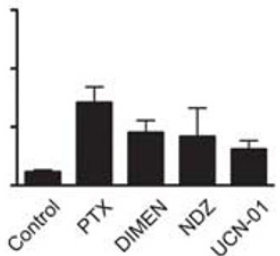

e

WT Diploid
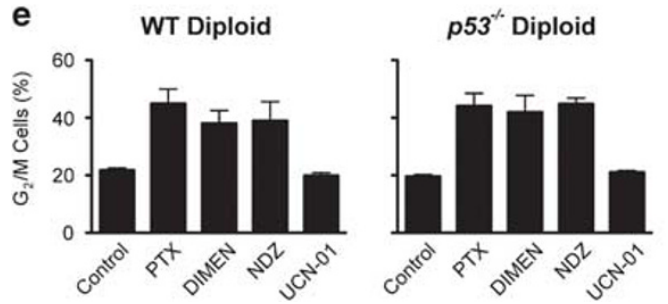

WT Tetraploid

$p 53^{-*}$ Tetraploid
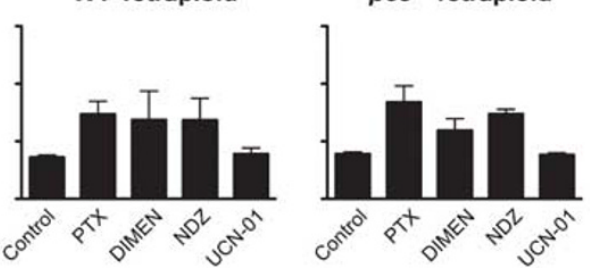

Figure 3 Automated detection of mitotic arrest. (a) Wild-type (WT) diploid HCT 116 cells were engineered for the co-expression of H2B-GFP and DsRed-Centrin (allowing for the visualization of chromatin and centrosomes, respectively). Fluorescence microphotographs of these cells in untreated conditions (Control) or after a $8 \mathrm{~h}$-long incubation with paclitaxel (PTX), dimethylenastron (DIMEN), nocodazole (NDZ), 7-hydroxystaurosporine (UCN-01) or staurosporine (STS) show that mitotic blockers (but not proapoptotic molecules) induced an increase in mitotic figures (scale bar $=10 \mu \mathrm{m}$ ). (b) PTX, DIMEN and NDZ (but not pro-apoptotic triggers) generated distinctive cell populations (corresponding to mitotic events) that localized in the quadrant defined by values higher than 550 and 250 arbitrary units (AUs) on a nuclear granularity versus nuclear mean densitometry plot, respectively. (c) Each mitotic blocker led to a specific nuclear morphology (namely, 'semicircular', 'circular' and 'solid' nuclei as induced by PTX, DIMEN and NDZ, respectively), which correlated with the distribution of nuclear granularity values (scale bar $=10 \mu \mathrm{m}$ ). (d) Fluorescence microphotographs were taken from WT diploid, $p 53^{-1-}$ diploid, WT tetraploid, $p 53^{-1-}$ tetraploid HCT 116 cells kept in untreated conditions (Control) or treated as in (a). Subsequent automated counting of events with nucleargranularity and nuclear mean densitometry higher than 550 and $250 \mathrm{AU}$, respectively, allowed for the quantification of cells in metaphase. (e) Fluorescence microscopy data were confirmed by the parallel assessment of cell cycle distribution by Hoechst 33342 staining and cytofluorometric quantification of cells with a $\mathrm{G}_{2} / \mathrm{M} \mathrm{DNA}$ content. Columns depict mean values \pm S.E.M. as recorded from three independent experiments. DsRed, Discosoma striata red fluorescent protein; GFP, green fluorescent protein; $\mathrm{H} 2 \mathrm{~B}$, histone $\mathrm{H} 2 \mathrm{~B}$

centrosomes within this ROI was determined using the DsRed-Centrin signal. Untreated WT diploid HCT 116 cells usually contained one or two (and only rarely three) red fluorescent dots (corresponding to centrosomes) (Figure 4a).
The fraction of surviving cells that harbored more than two centrosomes was consistently increased upon treatment with PTX, DIMEN and NDZ (but not UCN-01) (Figures 4b-e). Quantification of the nuclear area by fluorescence micro- 

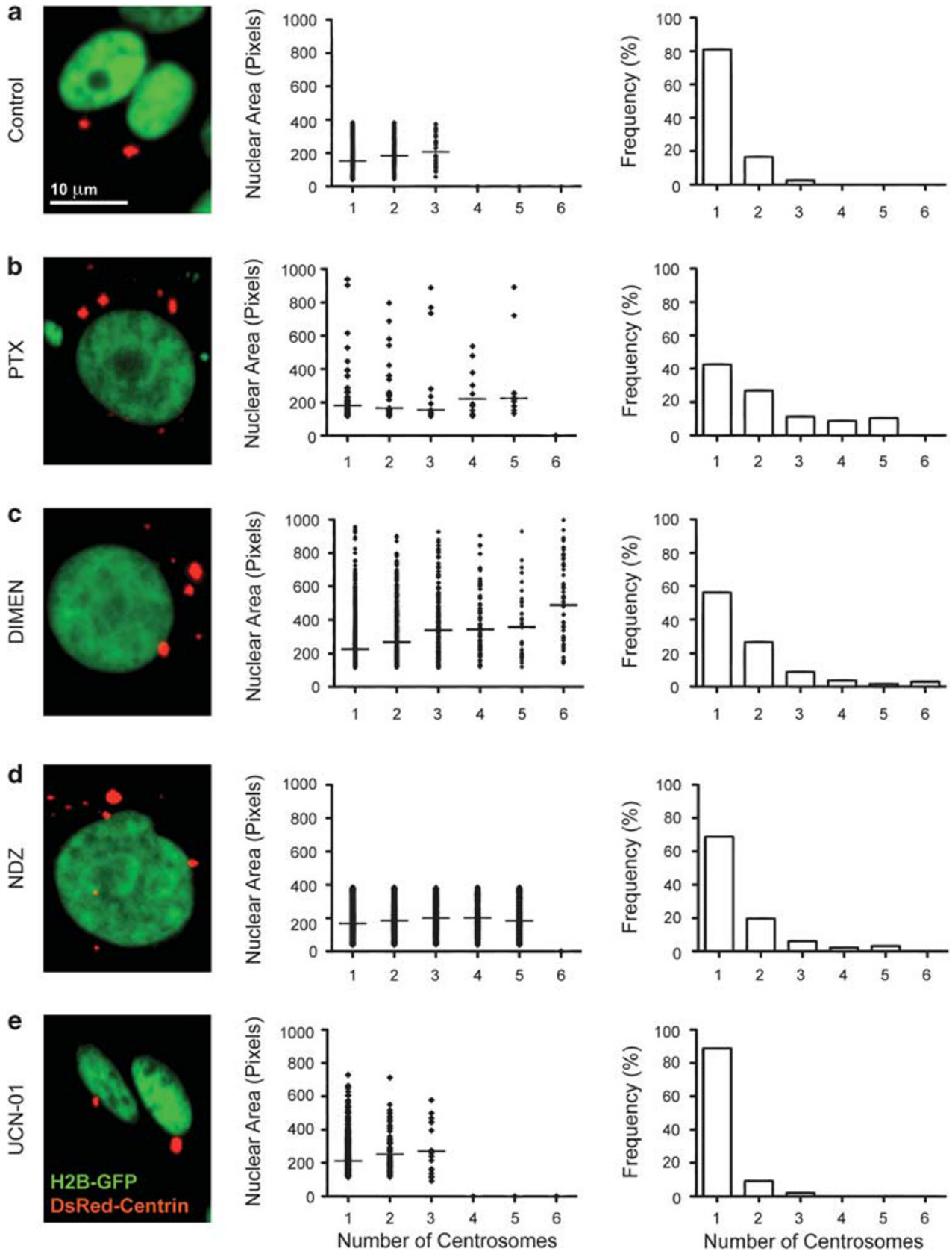

Figure 4 Mitotic blockers lead to increased nuclear area and to the generation of supernumerary centrosomes. Wild-type (WT) diploid HCT 116 cells co-expressing H2B-GFP and DsRed-Centrin (which allow for the monitoring of chromatin status and centrosome number, respectively) were left untreated (Control, a) or incubated with paclitaxel (PTX, b), dimethylenastron (DIMEN, c), nocodazole (NDZ, d), 7-hydroxystaurosporine (UCN-01, e) for $72 \mathrm{~h}$. Fluorescence microphotographs taken at this time point were then automatically analyzed for the quantification of nuclear area and the number of centrosomes per cell (within the surviving cell population). Microphotographs and quantitative data representative of one out of three independent experiments are shown (scale bar $=10 \mu \mathrm{m}$ ). DsRed, Discosoma striata red fluorescent protein; GFP, green fluorescent protein; $\mathrm{H} 2 \mathrm{~B}$, histone $\mathrm{H} 2 \mathrm{~B}$

scopy coupled to automated image analysis suggested that PTX, DIMEN and NDZ (but not UCN-01) augmented the median nuclear size of diploid and tetraploid cells, irrespective of their p53 status (Figures $4 a-e$ and 5a). The increment of nuclear area induced by mitotic blockers was slightly (yet significantly) smaller in tetraploid cells than in their diploid counterparts (Figure 5a). Polyploidization was corroborated by cytofluorometric analysis, showing that sub-populations of cells with an increased DNA content $\left(>G_{2} / M\right)$ were generated from diploid and tetraploid cells in response to mitotic blockers (Figure $5 b$ ). This finding is in accordance with reports on nuclear size constraints that preclude a direct correlation between DNA content and nuclear volume. ${ }^{45}$ Importantly, after the administration of PTX or DIMEN, $p 53^{-/}$diploid cells 

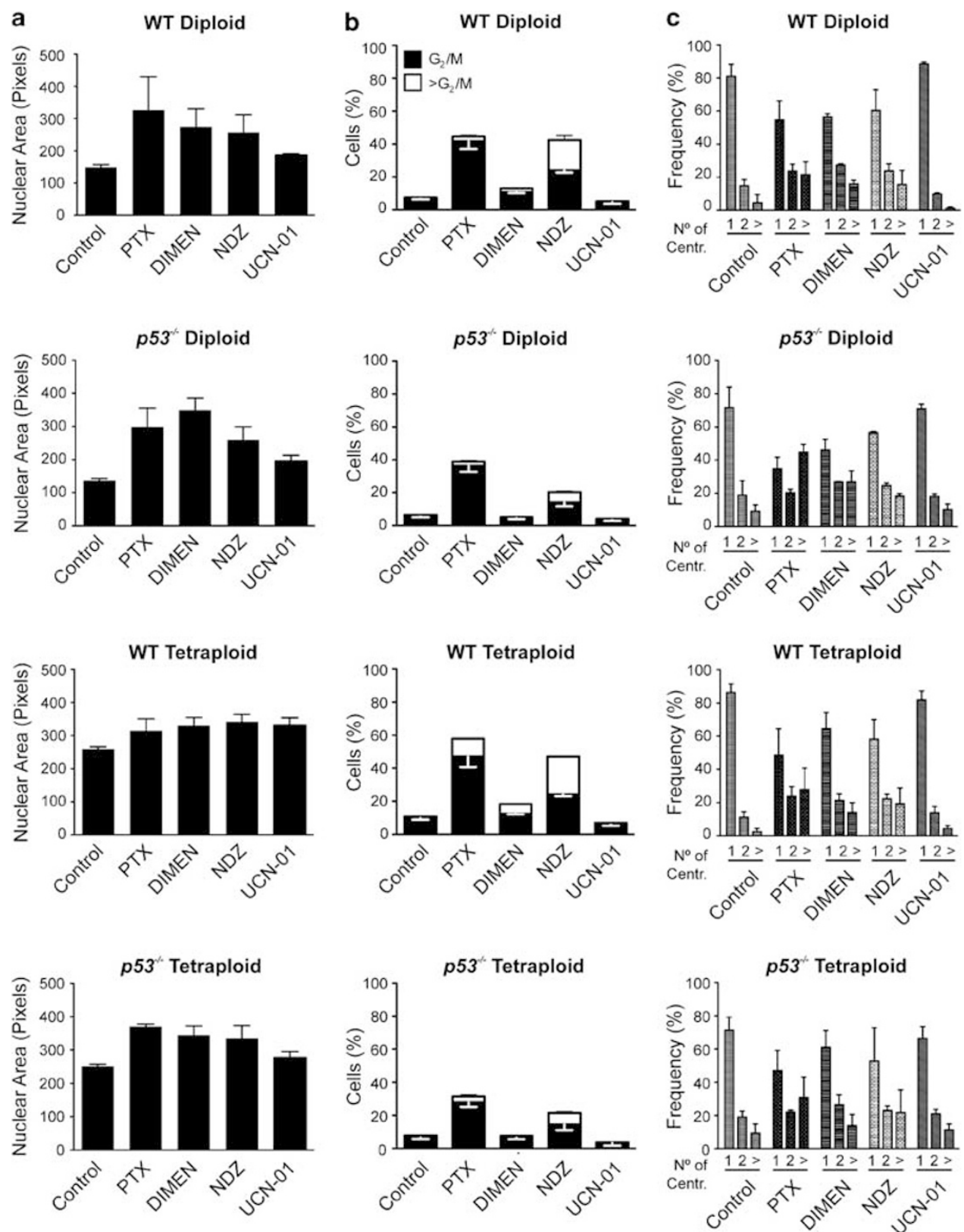

Figure 5 Automatic analysis of fluorescence microphotographs allows for the assessment of polyploidization. Human colon carcinoma HCT 116 cells with the indicated ploidy and p53 status stably expressing fusion proteins for the detection of chromatin status (H2B-GFP) and centrosome number (DsRed-Centrin) were left untreated (Control) or incubated with paclitaxel (PTX), dimethylenastron (DIMEN), nocodazole (NDZ) or 7-hydroxystaurosporine (UCN-01) for $72 \mathrm{~h}$. (a) Automated analysis of fluorescence microphotographs allowed for the quantification of nuclear area. Columns depict the mean of the median values \pm S.E.M., as recorded in three independent experiments. Mitotic blockers (but not UCN-01) provoked a relevant increase in the nuclear area of both diploid and tetraploid cells, irrespective of their p53 status. (b) Cytofluorometric analysis upon Hoechst 33342 staining confirmed that, in clear contrast to UCN-01, mitotic inhibitors (in particular PTX and NDZ) led to the accumulation of cells with a DNA content $\geqslant \mathrm{G}_{2} / \mathrm{M}$, in both diploid and tetraploid cells. As at this time point mitotic cells were not detectable by imaging (data not shown), these events represented bona fide aneuploid cells. (c) Automated quantification of the number of centrosomes per cell (No. of Centr.) suggested that $p 53^{-}{ }^{-}$HCT 116 cells tended to accumulate supernumerary centrosomes more readily than their wild-type (WT) counterparts. In (b) and (c), data are mean values \pm S.E.M. ( $n=3$ independent experiments)

were more prone to accumulate supernumerary centrosomes than their WT counterparts (Figure $5 \mathrm{c}$ ), which is in line with previous reports and confirms a role for p53 in the control of centrosome numbers. ${ }^{46}$ Altogether, these results indicate that fluorescence microscopy of adherent cells expressing specific biosensors can be used to detect supernumerary centrosomes and polyploidy in an automated manner.
Videomicroscopic detection of mitotic catastrophe. The quantitative assessments presented above were performed as end point assays on fixed cells, yet they are perfectly suitable for repeated measurements and kinetic determinations on living cells. Thus, we decided to track individual cells by fluorescence videomicroscopy to determine the time that would elapse between metaphase and the end 


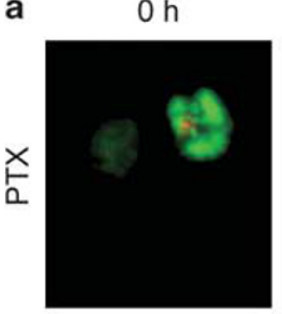

b

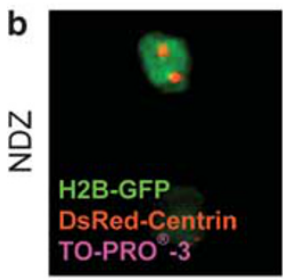

$6 \mathrm{~h}$
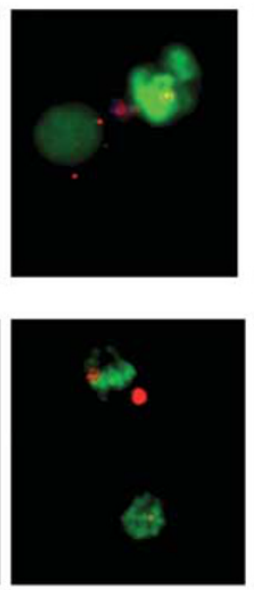

$12 \mathrm{~h}$
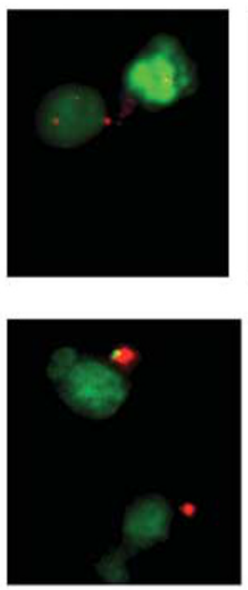

$22 \mathrm{~h}$
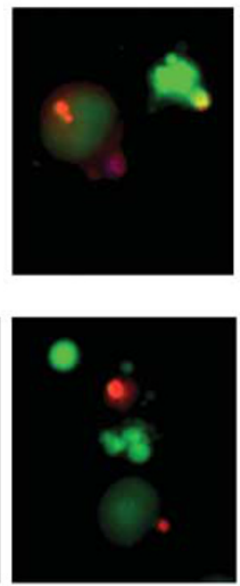

$24 \mathrm{~h}$
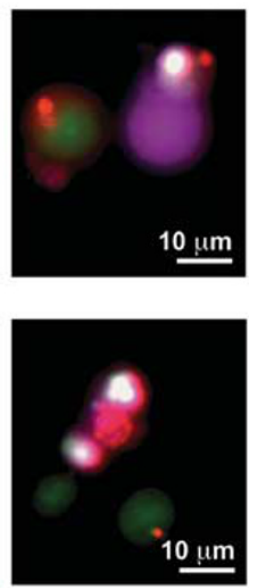

C Cell Fate Profile
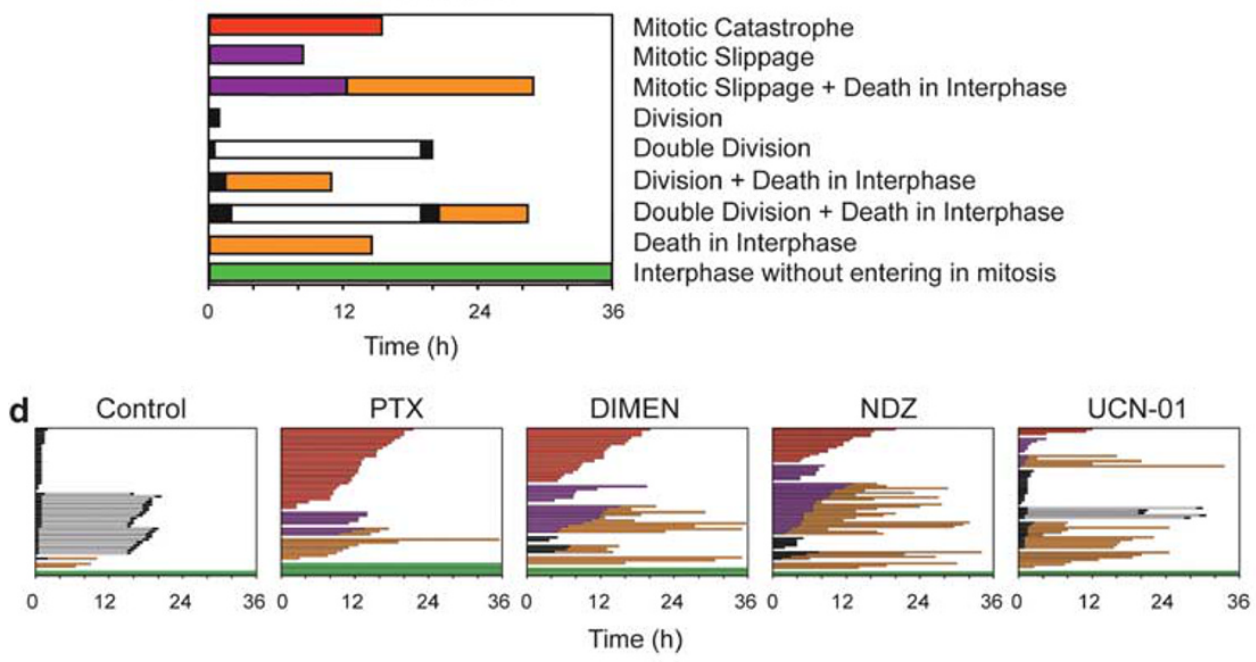

e

Control

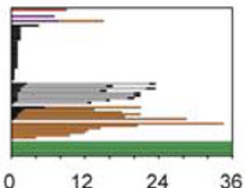

PTX

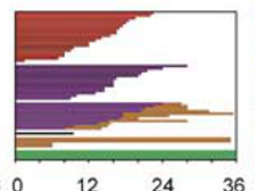

DIMEN

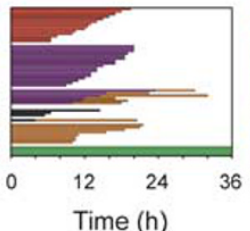

Mitotic Catastrophe

Mitotic Slippage

Mitotic Slippage + Death in Interphase

Division

Double Division

Division + Death in Interphase

Double Division + Death in Interphase

Death in Interphase

Interphase without entering in mitosis

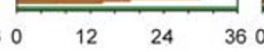

NDZ

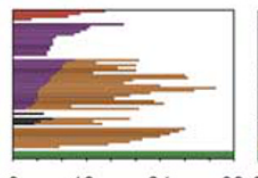

Figure 6 Time-lapse videomicroscopy of mitotic cell death in HCT 116 cells stably co-expressing DsRed-Centrin and H2B-GFP. Wild-type (WT) and p53 ${ }^{-1-}$ diploid human colon carcinoma HCT 116 cells stably co-expressing H2B-GFP and DsRed-Centrin (which allow for the visualization of chromatin and centrosomes, respectively) were left untreated (Control) or kept for $8 \mathrm{~h}$ in the presence of paclitaxel (PTX), dimethylenastron (DIMEN), nocodazole (NDZ) or 7-hydroxystaurosporine (UCN-01), and then monitored by videomicroscopy (upon addition of the vital dye TO-PRO-3) for the subsequent $36 \mathrm{~h}$ (still in the presence of the indicated drug). Please note that time 0 coincides with the beginning of videomicroscopic assessments ( $8 \mathrm{~h}$ after drug administration). (a) WT diploid cells treated with PTX show typical features of mitotic catastrophe: nuclei in metaphase directly undergo shrinkage well before TO-PRO-3 incorporation (which reflects plasma membrane permeabilization). (b) Upon NDZ administration, $p 53^{-/-}$diploid cells tended to show mitotic slippage to interphase before cell death. In this representative snapshot series, karyorrhexis occurred approximately $10 \mathrm{~h}$ after the recovery of interphase and preceded the uptake of TO-PRO-3. Scale bars $=10 \mu \mathrm{m}$. Full-length movies for (a) and (b) are available as Supplementary Videos 3 and 4 , respectively. (c) The fate of more than 50 single cells from at least three distinct movies was monitored by fluorescence videomicroscopy for up to $36 \mathrm{~h}$, cataloged and quantified according to the indicated events, which allowed for the generation of specific cell-fate profiles. Additional illustrations that depict the cataloged events and explain cell-fate diagrams are provided in Supplementary Figure 4. Thus, when compared with their WT counterparts (d), $p 53^{-1-}$ diploid cells (e) showed an increased resistance to mitotic catastrophe (as induced by PTX, DIMEN and NDZ) in association with a higher propensity to mitotic slippage. DsRed, Discosoma striata red fluorescent protein; GFP, green fluorescent protein; $\mathrm{H} 2 \mathrm{~B}$, histone $\mathrm{H} 2 \mathrm{~B}$ 
point phenotype. Several researchers have summarized the different outcomes of an abnormal mitosis, ${ }^{13,14,16,20}$ thereby defining death during mitosis (mitotic catastrophe, Figure 6a), reversion to interphase (mitotic slippage, Figure 6b) or completion of karyokinesis (division, Figure 1a), which can be followed by normal cell cycle progression, senescence or death with either apoptotic or necrotic features.

We started 36-h-long videomicroscopic acquisitions at $8 \mathrm{~h}$ after the addition of mitotic blockers (Figure 6) and classified the observed cellular fates in nine different categories: (1) mitotic catastrophe, (2) mitotic slippage followed by interphase, (3) mitotic slippage followed by death in interphase, (4) division yielding viable daughter cells, (5) double division (daughter cells also divided during the experiment), (6) division followed by death in interphase, (7) double division followed by death in interphase, (8) cell death in interphase (in the absence of any mitotic event) and (9) prolonged interphase (in the absence of any mitotic or cell death event) (Supplementary Figure 4 and Figure 6c). Our system does not allow for the detection of senescence, as a much longer (theoretically infinite) video recording time would be required for this, nor for the discrimination between karyokinesis and cytokinesis.

In spite of these limitations, this videomicroscopic setup allowed us to generate cell-fate profiles for WT and $p 53^{-1-}$ diploid cells (Figures $6 d$ and e). Thus, WT diploid cells treated with PTX, DIMEN and NDZ massively underwent mitotic catastrophe (Figure 6d, red bars), yet also showed some extent of cell death during interphase (Figure $6 \mathrm{~d}$, orange bars). The same did not occur upon the administration of UCN-01, which was associated with very few instances of mitotic cell death (Figure $6 \mathrm{~d}$ ). After the administration of mitotic blockers, the fraction of $p 53^{-/}$diploid cells that underwent mitotic catastrophe was lower than that observed among their WT counterparts, as can be appreciated by comparing the amount of red bars in Figures $6 \mathrm{~d}$ and e. In these conditions, $p 53^{-1-}$ cells rather reverted to interphase and eventually survived (Figures $6 \mathrm{~d}$ and e, violet bars), in line with the notion that the absence of p53 facilitates mitotic slippage. ${ }^{16,47}$ In both p53-proficient and p53-deficient diploid cells, there was a linear correlation between the frequency of cells bearing supernumerary centrosomes and that of mitotic catastrophes (Figure 7). However, the slope of the linear regression between these two data sets was higher in $p 53^{-/}$ cells than in their WT counterparts, in line with the conclusion that the former generate and tolerate a higher number of extra centrosomes than the latter. Altogether, these experiments support the possibility to quantify mitotic catastrophe by videomicroscopy coupled to automated imaging.

\section{Materials and Methods}

Reagents, cell lines, culture conditions and treatments. Unless otherwise indicated, chemicals were obtained from Sigma-Aldrich (Saint Louis, MO, USA). Media and supplements for cell culture were purchased from Gibco-Invitrogen (Carlsbad, CA, USA). Plasticware was obtained from Corning B.V. Life Sciences (Schiphol-Rijk, The Netherlands).

WT and $p 53^{-1-}$ human colon carcinoma HCT 116 cells (kind gift from Bert Vogelstein $)^{38}$ were routinely maintained in McCoy's $5 \mathrm{~A}$ medium supplemented with $10 \%$ fetal bovine serum (FBS) and $10 \mathrm{mM} \mathrm{HEPES}$ buffer (at $37^{\circ} \mathrm{C}$ in a $5 \% \mathrm{CO}_{2}$ atmosphere). Tetraploid cell lines were generated by transient ( $48 \mathrm{~h}$ ) exposure to $100 \mathrm{nM}$ NDZ followed by fluorescence-activated cell sorter (FACS) purification of a

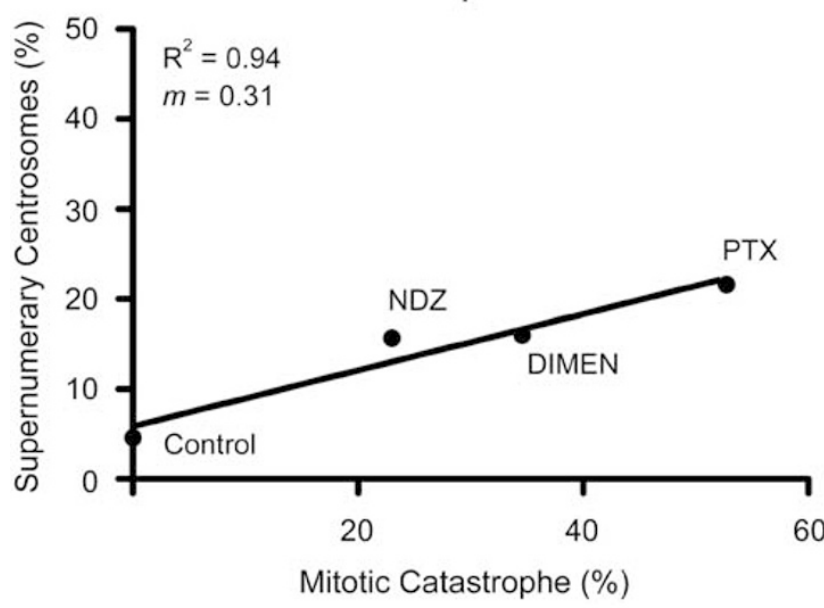

b

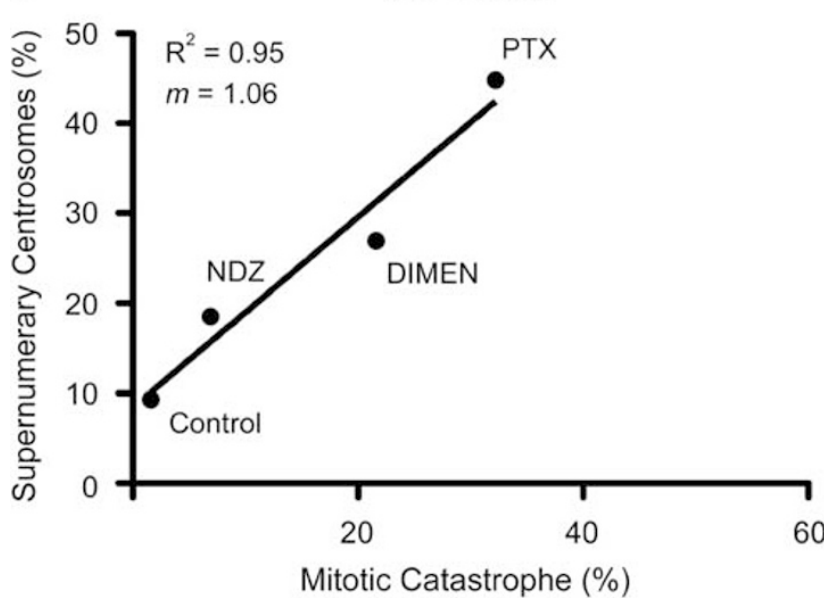

Figure 7 Linear correlation between the percentage of cells with supernumerary centrosomes and the incidence of mitotic catastrophe. Wild-type (WT) and $\mathrm{p} 53^{-1-}$ diploid human colon carcinoma HCT 116 cells that constitutively co-express biosensors for the detection of chromatin status and centrosomes were cultured for $8 \mathrm{~h}$ in the absence (Control) or in the presence of paclitaxel (PTX), dimethylenastron (DIMEN), nocodazole (NDZ) or 7-hydroxystaurosporine (UCN-01), and then monitored by videomicroscopy for the automated quantification of the number of centrosomes per cell and of mitotic catastrophe. In both WT (a) and p53 $3^{-1-}$ (b) diploid cells, the percentage of cells characterized by supernumerary centrosomes linearly correlated with the incidence of mitotic catastrophe. Data are mean values from three independent experiments. $\mathrm{m}=$ slope; $R^{2}=$ coefficient of determination

cells with an $\sim 8 \mathrm{c}$ DNA content (which are bona fide tetraploid cells in $G_{2} / M$ ), as previously described (Supplementary Figure 1). ${ }^{16,39}$ Human osteosarcoma U2OS cells were cultured in Dulbecco's modified Eagle's medium (DMEM) supplemented with $10 \%$ FBS, $1 \mathrm{mM}$ sodium pyruvate and $10 \mathrm{mM}$ HEPES buffer.

To induce mitotic catastrophe, HCT 116 cells were administered with $1 \mu \mathrm{M}$ DIMEN(Calbiochem-Merck, Darmstadt, Germany), $500 \mathrm{nM}$ PTX or $100 \mathrm{nM}$ NDZ. Alternatively, apoptosis was triggered by $500 \mathrm{nM}$ STS or by the same concentration of its derivative UCN-01. U2OS cells were treated with $4 \mu \mathrm{M}$ DIMEN, $1.5 \mu \mathrm{M}$ PTX, $300 \mathrm{nM}$ NDZ or $1.5 \mu \mathrm{M}$ UCN-01. Stock solutions were prepared following the manufacturers' instructions.

Stable transfection with DsRed-centrin. A cDNA encoding for centrin derived from a human testis mRNA library (GenBank accession number 
BC029515.1) was amplified in a pCMV-sport6 vector (Invitrogen, Eugene, OR, USA) and then cloned into a pDsRed2-C1 plasmid (Clontech, Mountain View, CA, USA) as a Bg/ll restriction fragment (Supplementary Figure 2). HCT 116 cells stably expressing H2B-GFP fusion protein (which allows for the visualization of chromatin) ${ }^{31,39}$ were co-transfected with this DsRed-Centrin-coding construct plus pPUR (Clontech), which confers puromycin resistance (required for the selection of transfectants as $p 53^{-/-}$HCT 116 cells are constitutively resistant to both neomycin and hygromycin). ${ }^{38}$ Stable clones were generated by limiting dilution and validated by fluorescence microscopy. After selection, the growth rates of transfected cells were quantified by hemocytometric counting (Hycor, Garden Grove, CA, USA) upon Trypan blue staining, and compared with those of their untransfected counterparts.

Videomicroscopy and end point image acquisition. At 1 day before the experiments, $1 \times 10^{4}$ cells were seeded in 96-well Black/Clear Imaging Plates (BD Biosciences, San Jose, CA, USA) pre-treated with poly-L-lysine. Chemicals were administered approximately $8 \mathrm{~h}$ before live cell imaging on a BD Pathway 855 High-Content Biolmager workstation (BD Biosciences) equipped with Photofluor light sources (Chroma, Rockingham, VT, USA), different excitation and emission filters (Semrock, Rochester, NY, USA) and a ORCA-AG Deep Cooled Digital Camera (Hamamatsu Photonics, Hamamatsu, Japan).

For the assessment of plasma membrane integrity $1 \mu \mathrm{M}$ TO-PRO-3 (Molecular Probes-Invitrogen) was used in time-lapse microscopy. Images were captured every 30 min with the appropriate settings for GFP (488/10 excitation, 515LP emission filters, 50 ms exposure, 25 gain, 215 offset), DsRed (548/20 excitation, 570LP emission filters, $22 \mathrm{~ms}$ exposure, 15 gain, 210 offset) and TO-PRO-3 (635/20 excitation, 84101 emission filters, $10 \mathrm{~ms}$ exposure, 30 gain, 219 offset) fluorescence with an UApo/340 $\times 40 / 0.90$ objective (Olympus, Tokyo, Japan). Movies were then processed using BD AttoVision (BD Biosciences) and ImageJ (freely available from the National Institute of Health, Bethesda, MD, USA at http://rsb.info.nih.gov/ij/) software. ${ }^{30}$

For end point analysis (8, 24, 48 and $72 \mathrm{~h}$ after treatment), cells were fixed for $15 \mathrm{~min}$ in cold $4 \%(\mathrm{v} / \mathrm{v})$ formaldehyde and subsequently imaged on the BD Pathway 855 High-Content Biolmager workstation using an UApo/340 $\times 20 / 0.75$ objective (Olympus) and an Infinity camera (Lumenera, Ottawa, ON, Canada). These settings were applied for the acquisition of GFP (482/35 excitation, 536/40 emission filters, $11 \mathrm{~ms}$ exposure, 0 gain) and DsRed (confocal mode, 543/22 excitation, 593/40 emission filters, 800 ms exposure, 8 gain, collapsed stack of 7 sections of $1.429 \mu \mathrm{m}$ each in a whole height of $10 \mu \mathrm{m}$ ) signals.

Image segmentation. Upon image acquisition, the BD AttoVision software was used to segment the ROls for subsequent analysis: nuclei (identified using H2B-GFP signal from chromatin), cells (defined by the nuclear area plus a perinuclear extension of 10 pixels) and centrosomes (monitored using DsRedCentrin signal) (Supplementary Figure 4). The objects were equalized and contrasted from the background by means of shading and rolling-ball preprocessing filters ( $75 \times 75$ for nuclei and $2 \times 2$ for centrosomes), before automatic signal-to-noise thresholding.

To exclude from the analysis events corresponding to more than one nucleus, successful segregation of each nucleus as a unique ROI was conducted using an open pre-processing filter combined with a watershed erosion algorithm. Cellular debris were left out by applying a 'scrap' algorithm set to a minimum size limit of 100 pixels, whereas the possible bias coming from overlapping nuclei clusters was eluded by introducing a maximum size limit value for images characterized by high cellular density.

Acquisition of quantitative parameters. In segmented images, the number of centrosomes found in each cell was quantified by sub-object count, in parallel with the assessment of nuclear area, nuclear 'granularity' and nuclear 'mean densitometry'. Cell ROls apparently lacking the DsRed-Centrin signal were excluded from subsequent analytical procedures. The number of pixels required for nuclear ROI segmentation defined the nuclear area. Granularity was evaluated by comparing the average difference of fluorescence intensity of adjacent pixels within a ROI to the mean fluorescence intensity of the entire ROI. This parameter measures the presence of adjacent zones showing heterogeneous fluorescence (e.g., as occurring during mitosis or apoptosis, both of which are associated with chromatin condensation), and hence allow for the detection/quantification of changes in the distribution of the fluorescent signal from H2B-GFP (Supplementary Figure 3). Mean densitometry was calculated as the ratio between the average fluorescence intensity of the ROI and its area. Statistical data treatment was performed using the BD Image Data Explorer software (BD Biosciences). Eventually, mitotic cells were defined by granularity of $>550$ AUs and mean densitometry of $>250$ AUs.

Cytofluorometric analyses. For the simultaneous quantification of DNA content and plasma membrane integrity, cells were co-stained with the DNA intercalating agent Hoechst $33342(10 \mu \mathrm{g} / \mathrm{ml}$, Molecular Probes-Invitrogen) and $1 \mu \mathrm{g} / \mathrm{ml}$ propidium iodide ( $\mathrm{PI}$, an exclusion dye that at this concentration is incorporated only in dead cells) for $30 \mathrm{~min}$ at $37^{\circ} \mathrm{C} .{ }^{48-50}$ Cytofluorometric acquisition of Hoechst 33342 and PI fluorescence was carried out using a FACS Vantage (BD Biosciences) equipped with a $70 \mu \mathrm{m}$ nozzle. Exclusively events characterized by standard forward scatter (FSC) and side scatter (SSC) parameters were included in subsequent analyses. DNA content studies were limited to living cells by gating on the $\mathrm{PI}^{-}$cell population. Data were evaluated using the CellQuest software (BD Biosciences).

Cell proliferation assays. Cell proliferation was quantified using a colorimetric assay based on the reduction of the colorless tetrazolium salt 4-[3-(4iodophenyl)-2-(4-nitrophenyl)-2H-5-tetrazolio]-1,3-benzene disulfonate (WST-1; Roche, Penzberg, Germany) to formazan (which shows an absorbance peak of approximately $450 \mathrm{~nm}$ ), according to the manufacturer's instructions. Absorbance was measured in a Dynex MRX II microplate reader (Magellan Biosciences, Chelmsford, MA, USA).

\section{Concluding Remarks}

Mitotic catastrophe is a cell death modality that is linked to morphological, and more importantly, 'historical' features of the cell, thereby requiring a specialized method of detection. Indeed, conventional biochemical bulk assays (such as caspase activation tests) cannot discriminate between interphase and post-mitotic apoptosis. Similarly, cytofluorometric methods cannot determine whether apoptotic traits (such as phosphatidylserine exposure or the collapse of the mitochondrial transmembrane potential) have been acquired before, during or after mitosis. Thus, to precisely identify mitotic catastrophe, additional morphological changes have to be monitored, ideally in a way that allows for the assessment of the fate of individual cells in the context of mitotic aberrations.

In this study we have improved a set of experimental procedures and software settings that permits the quantification of mitosis, ploidy, supernumerary centrosomes and apoptotic cell death by fluorescence (video)microscopy coupled to automated image analysis. Most of the results presented in this article were generated in HCT 116 cells, a model of human colon carcinoma that allowed us to study the influence of p53 in the outcomes of mitotic catastrophe. Nonetheless, our experimental procedures are fully suitable to other cell lines (such as human osteosarcoma U2OS cells, Supplementary Figure 5) that tolerate the expression of the chimeric proteins that we used. Image segmentation as well as texture and object-counting analyses can be achieved by videomicroscopes and software (e.g., ImageJ, freely available at http://rsb.info.nih.gov/ij/; Metamorph, from Molecular Devices, Sunnyvale, CA, USA) that are distinct from those used in this paper. Thus, our experimental and analytical procedures can be adapted to platforms from different commercial providers.

In summary, when combined with kinetic assessments, these methods promise to facilitate the automatic detection of mitosis-related cell death and hence to constitute an objective method for the quantification of mitotic catastrophe.

\section{Conflict of interest}

The authors declare no conflict of interest.

Acknowledgements. We thank Verónica Labrador Catarero from the Optical and Confocal Microscopy Facility of Centro de Biología Molecular Severo Ochoa (Madrid, Spain) for helpful discussion. GK is supported by the Ligue Nationale contre le Cancer (Equipe labellisée), Agence Nationale pour la Recherche, European Commission (Apo-Sys, ChemoRes, ApopTrain), Fondation pour la Recherche Médicale, Institut National du Cancer, Cancéropôle lle-de-France. SR-V, MM and LS are supported by FRM. OK is supported by EMBO. LG is supported by the Apo-Sys consortium of the European Union. MC is supported by the Association pour la Recherche sur le Cancer (ARC). 
1. Kroemer G, Galluzzi L, Brenner C. Mitochondrial membrane permeabilization in cell death. Physiol Rev 2007; 87: 99-163.

2. Galluzzi L, Morselli E, Kepp O, Kroemer G. Targeting post-mitochondrial effectors of apoptosis for neuroprotection. Biochim Biophys Acta 2009; 1787: 402-413.

3. Galluzzi L, Blomgren K, Kroemer G. Mitochondrial membrane permeabilization in neuronal injury. Nat Rev Neurosci 2009; 10: 481-494.

4. Galluzzi L, Larochette N, Zamzami N, Kroemer G. Mitochondria as therapeutic targets for cancer chemotherapy. Oncogene 2006; 25: 4812-4830.

5. Kroemer G, Galluzzi L, Vandenabeele P, Abrams J, Alnemri ES, Baehrecke EH et al. Classification of cell death: recommendations of the Nomenclature Committee on Cell Death 2009. Cell Death Differ 2009; 16: 3-11.

6. Galluzzi L, Vicencio JM, Kepp O, Tasdemir E, Maiuri MC, Kroemer G. To die or not to die: that is the autophagic question. Curr Mol Med 2008; 8: 78-91.

7. Galluzzi L, Aaronson SA, Abrams J, Alnemri ES, Andrews DW, Baehrecke EH et al. Guidelines for the use and interpretation of assays for monitoring cell death in higher eukaryotes. Cell Death Differ 2009; 16: 1093-1107.

8. Klionsky DJ, Abeliovich H, Agostinis P, Agrawal DK, Aliev G, Askew DS et al. Guidelines for the use and interpretation of assays for monitoring autophagy in higher eukaryotes. Autophagy 2008; 4: 151-175.

9. Tasdemir E, Galluzzi L, Maiuri MC, Criollo A, Vitale I, Hangen E et al. Methods for assessing autophagy and autophagic cell death. Methods Mol Biol 2008; 445: 29-76.

10. Roninson IB, Broude EV, Chang BD. If not apoptosis, then what? Treatment-induced senescence and mitotic catastrophe in tumor cells. Drug Resist Updat 2001; 4: 303-313.

11. Suzuki K, Ojima M, Kodama S, Watanabe M. Delayed activation of DNA damage checkpoint and radiation-induced genomic instability. Mutat Res 2006; 597: 73-77.

12. Castedo M, Perfettini JL, Roumier T, Valent A, Raslova H, Yakushijin $\mathrm{K}$ et al. Mitotic catastrophe constitutes a special case of apoptosis whose suppression entails aneuploidy. Oncogene 2004; 23: 4362-4370.

13. Vakifahmetoglu $\mathrm{H}$, Olsson M, Zhivotovsky B. Death through a tragedy: mitotic catastrophe. Cell Death Differ 2008; 15: 1153-1162.

14. Weaver BA, Cleveland DW. Decoding the links between mitosis, cancer, and chemotherapy: the mitotic checkpoint, adaptation, and cell death. Cancer Cell 2005; 8 : 7-12.

15. Castedo M, Perfettini JL, Roumier T, Andreau K, Medema R, Kroemer G. Cell death by mitotic catastrophe: a molecular definition. Oncogene 2004; 23: 2825-2837.

16. Castedo M, Coquelle A, Vivet S, Vitale I, Kauffmann A, Dessen P et al. Apoptosis regulation in tetraploid cancer cells. EMBO J 2006; 25: 2584-2595.

17. Mansilla S, Bataller M, Portugal J. Mitotic catastrophe as a consequence of chemotherapy Anticancer Agents Med Chem 2006; 6: 589-602.

18. Sundaram M, Guernsey DL, Rajaraman MM, Rajaraman R. Neosis: a novel type of cell division in cancer. Cancer Biol Ther 2004; 3: 207-218.

19. Vitale I, Senovilla L, Jemaa M, Michaud M, Galluzzi L, Kepp $O$ et al. Multipolar mitosis of tetraploid cells leads to pseudo-diploidy in a Mos-dependent fashion. EMBO J2010 (in press)

20. Erenpreisa J, Cragg MS. Cancer: a matter of life cycle? Cell Biol Int 2007; 31: 1507-1510.

21. Cristobal J, Stockert JC, Villanueva A, Rello-Varona S, Juarranz A, Canete M. Caspase-2 a possible trigger of apoptosis induced in A-549 tumor cells by $\mathrm{ZnPc}$ photodynamic treatment. Int J Oncol 2006; 28: 1057-1063.

22. Krumschnabel G, Sohm B, Bock F, Manzl C, Villunger A. The enigma of caspase-2: the laymen's view. Cell Death Differ 2009; 16: 195-207.

23. Olsson M, Vakifahmetoglu H, Abruzzo PM, Hogstrand K, Grandien A, Zhivotovsky B. DISC-mediated activation of caspase-2 in DNA damage-induced apoptosis. Oncogene 2009; 28: 1949-1959.

24. Tu S, McStay GP, Boucher LM, Mak T, Beere HM, Green DR. In situ trapping of activated initiator caspases reveals a role for caspase-2 in heat shock-induced apoptosis. Nat Cell Biol 2006; 8: 72-77.

25. Vakifahmetoglu H, Olsson M, Tamm C, Heidari N, Orrenius S, Zhivotovsky B. DNA damage induces two distinct modes of cell death in ovarian carcinomas. Cell Death Differ 2008; 15: $555-566$.

26. Ganem NJ, Pellman D. Limiting the proliferation of polyploid cells. Cell 2007; 131: 437-440.

27. Rello-Varona S, Gamez A, Moreno V, Stockert JC, Cristobal J, Pacheco M et al. Metaphase arrest and cell death induced by etoposide on HeLa cells. Int J Biochem Cell Biol 2006; 38: 2183-2195.
28. Tao $\mathrm{Y}$, Zhang $\mathrm{P}$, Girdler $\mathrm{F}$, Frascogna V, Castedo $\mathrm{M}$, Bourhis $\mathrm{J}$ et al. Enhancement of radiation response in $\mathrm{p53}$-deficient cancer cells by the Aurora-B kinase inhibitor AZD1152. Oncogene 2008; 27: 3244-3255.

29. Cenciarelli C, Tanzarella C, Vitale I, Pisano C, Crateri P, Meschini S et al. The tubulindepolymerising agent combretastatin-4 induces ectopic aster assembly and mitotic catastrophe in lung cancer cells H460. Apoptosis 2008; 13: 659-669.

30. Rello-Varona S, Vitale I, Kepp O, Senovilla L, Jemaa M, Metivier D et al. Preferential killing of tetraploid tumor cells by targeting the mitotic kinesin Eg5. Cell Cycle 2009; 8: 1030-1035.

31. Vitale I, Galluzzi L, Vivet S, Nanty L, Dessen P, Senovilla L et al. Inhibition of Chk1 kills tetraploid tumor cells through a p53-dependent pathway. PLOS ONE 2007; 2: e1337.

32. Gascoigne KE, Taylor SS. Cancer cells display profound intra- and interline variation following prolonged exposure to antimitotic drugs. Cancer Cell 2008; 14: 111-122.

33. Golstein P, Kroemer G. Cell death by necrosis: towards a molecular definition. Trends Biochem Sci 2007; 32: 37-43.

34. Galluzzi L, Kroemer G. Necroptosis: a specialized pathway of programmed necrosis. Cell 2008; 135: 1161-1163.

35. Garrido C, Galluzzi L, Brunet M, Puig PE, Didelot C, Kroemer G. Mechanisms of cytochrome c release from mitochondria. Cell Death Differ 2006; 13: 1423-1433.

36. Glory E, Murphy RF. Automated subcellular location determination and high-throughput microscopy. Dev Cell 2007; 12: 7-16.

37. De Vos WH, Van Neste L, Dieriks B, Joss GH, Van Oostveldt P. High content image cytometry in the context of subnuclear organization. Cytom Part A 2010; 77: 64-75.

38. Bunz F, Dutriaux A, Lengauer C, Waldman T, Zhou S, Brown JP et al. Requirement for p53 and p21 to sustain G2 arrest after DNA damage. Science 1998; 282: 1497-1501.

39. Castedo M, Coquelle A, Vitale I, Vivet S, Mouhamad S, Viaud S et al. Selective resistance of tetraploid cancer cells against DNA damage-induced apoptosis. Ann NY Acad Sci 2006; 1090: 35-49.

40. Senovilla L, Vitale I, Galluzzi L, Vivet S, Joza N, Younes AB et al. p53 represses the polyploidization of primary mammary epithelial cells by activating apoptosis. Cell Cycle 2009; 8: 1380-1385.

41. Jordan MA, Toso RJ, Thrower D, Wilson L. Mechanism of mitotic block and inhibition of cell proliferation by taxol at low concentrations. Proc Natl Acad Sci USA 1993; 90: 9552-9556.

42. Hamilton BT, Snyder JA. Rapid completion of mitosis and cytokinesis in PtK cells following release from nocodazole arrest. Eur J Cell Biol 1982; 28: 190-194.

43. Ruegg UT, Burgess GM. Staurosporine, K-252 and UCN-01: potent but nonspecific inhibitors of protein kinases. Trends Pharmacol Sci 1989; 10: 218-220.

44. Ganem NJ, Godinho SA, Pellman D. A mechanism linking extra centrosomes to chromosomal instability. Nature 2009; 460: 278-282.

45. Webster M, Witkin KL, Cohen-Fix O. Sizing up the nucleus: nuclear shape, size and nuclear-envelope assembly. J Cell Sci 2009; 122: 1477-1486.

46. Fukasawa K. P53, cyclin-dependent kinase and abnormal amplification of centrosomes. Biochim Biophys Acta 2008; 1786: 15-23.

47. Meek DW. The role of $p 53$ in the response to mitotic spindle damage. Pathol Biol (Paris) 2000; 48: 246-254.

48. Castedo M, Ferri K, Roumier T, Metivier D, Zamzami N, Kroemer G. Quantitation of mitochondrial alterations associated with apoptosis. J Immunol Methods 2002; 265: 39-47.

49. Galluzzi L, Zamzami N, de La Motte Rouge T, Lemaire C, Brenner C, Kroemer G. Methods for the assessment of mitochondrial membrane permeabilization in apoptosis. Apoptosis 2007; 12: 803-813.

50. Galluzzi L, Vitale I, Kepp O, Seror C, Hangen E, Perfettini JL et al. Methods to dissect mitochondrial membrane permeabilization in the course of apoptosis. Methods Enzymol 2008; 442: 355-374.

Cell Death and Disease is an open-access journal published by Nature Publishing Group. This article is licensed under a Creative Commons Attribution-Noncommercial-No Derivative Works 3.0 License. To view a copy of this license, visit http:// creativecommons.org/licenses/by-nc-nd/3.0/

\section{Supplementary Information accompanies the paper on Cell Death and Disease website (http://www.nature.com/cddis)}

